\title{
Atmospheric calibration for submillimeter and terahertz observations
}

\author{
Xin Guan ${ }^{1}$, Jürgen Stutzki ${ }^{1}$ and Yoko Okada ${ }^{1}$ \\ ${ }^{1}$ I. Physikalisches Institut der Universität zu Köln, \\ Zülpicher Straße 77, 50937 Köln, Germany \\ email: guan@ph1.uni-koeln.de
}

\begin{abstract}
Submillimeter observations through the atmosphere can be affected by the complex spectroscopic features of the air. Calibration of astronomical observations in these frequencies requires proper modelling of the atmosphere. We analyzed sky observations from altitudes around 500 and $200 \mathrm{hPa}$ respectively and found deficiencies in atmospheric models. Further research to improve the models are expected to help in future submillimeter observations.
\end{abstract}

Keywords. Atmospheric effects - Submillimeter: general

The earth's atmosphere causes significant attenuation to submillimeter radio signals. For this reason, submillimeter astronomical observations must be carried out either from space or from high altitudes where the preciptable water vapour is low.

Observing from spacecraft is an expensive task. Particularly, when the apparatus needs frequent maintenance or is simply too large to be launched. Then we must make use of ground based, airborne or balloon-borne observatories. For the first two options, atmospheric calibration can be essential to getting accurate scientific data.

When observing with a double-side-banded (DSB) receiver such as the GREAT (Heyminck et al. (2012)) and the SMART (Graf et al. (2003)) receivers, an atmospheric model (atm-model hereafter) is necessary to separate the contributions of the atmosphere from each sideband. Utilization of an atm-model can also improve signal-to-noise (s/n) ratio for spectroscopy because the $\mathrm{s} / \mathrm{n}$ ratio directly obtained from limited observing time can be very low in the narrow frequency channels.

In order to model the atmosphere, firstly we modeled the vertical water vapour distribution (Guan et al. (2012)) according to meteorological measurements. Effective observation in submillimeter wavelengths requires very low precipitable water vapor (pwv). So the water mixing ratio is negligible. Therefore we can keep other parameters constant while varying the pwv, and prove that in our model the atmospheric opacity can be decomposited into wet and dry components (see Guan et al. (2012)):

$$
\tau=b \times p w v+c .
$$

Using Kirchhoff's law we can derive the atmosphere's opacity from its measured brightness temperature and then obtain pwv, the model parameter.

We made observations with the GREAT receiver on the SOFIA telescope from altitudes from 10 to $14 \mathrm{~km}$, and with the SMART receiver on the NANTEN2 telescope at an altitude of $4.8 \mathrm{~km}$. GREAT receives in several bands from 1.2 to $2.8 \mathrm{THz}$, while SMART is an array receiver of two bands $(460 / 490 \mathrm{GHZ}$ and $810 \mathrm{GHz})$ with 8 pixels each. SMART's unique property is that it is an array receiver and the results from its eight pairs of pixels can be cross-checked. The same cannot be easily done with GREAT/SOFIA.

Both of our GREAT and SMART observations showed inconsistencies with the atmmodel. The fit model parameter "pwv" is often inconsistent between different bands. 

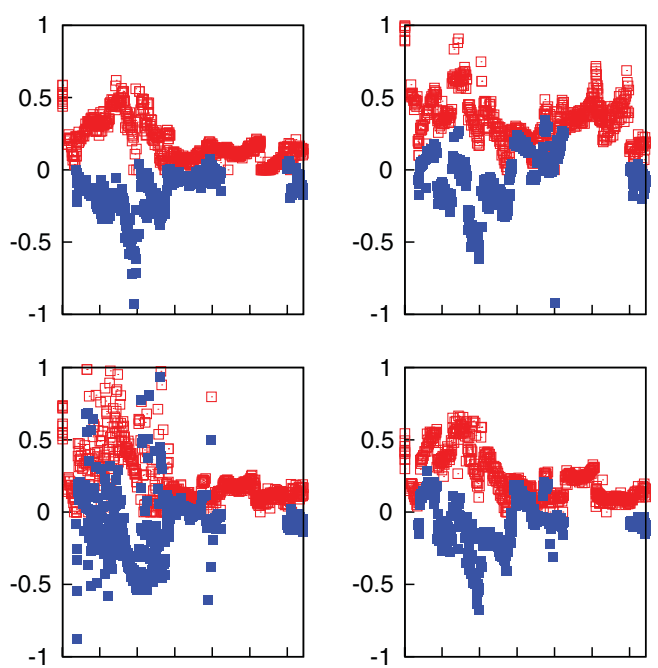
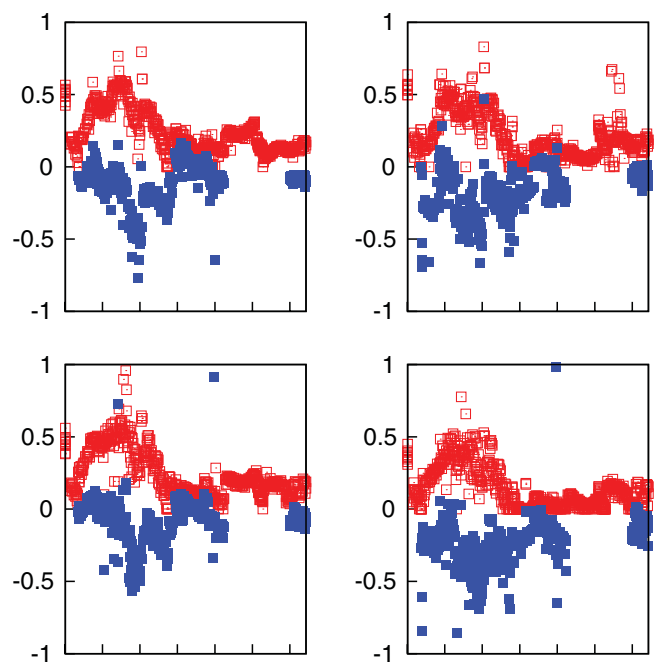

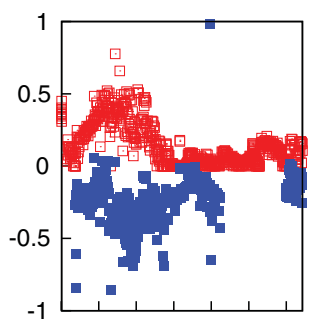

Figure 1. Fit pwv and dry-offset of SMART observations across 630 successive scans, in which pwv varied from a little below $1 \mathrm{~mm}$ to somewhere between 0.1 and $0.2 \mathrm{~mm}$. "Common-fit" was used to fit these spectra so there is only a single pwv and dry-offset for each $460 / 810 \mathrm{GHz}$ pairs. Red open squares are pwv's (millimeter) and blue filled squares are dry-offsets (neper). The horizontal axis is scan number. The eight subfigures show results from the eight pairs of $460 / 810 \mathrm{GHz}$ pixels at corresponding positions in the focal plane.

Trying to find out the problem, we introduced the "dry-offset" parameter to the model:

$$
\tau_{\nu}=b_{\nu} p w v+c_{\nu}- \begin{cases}o_{L 1}, & \nu \text { in L1 } \\ o_{L 2}, & \nu \text { in L2 }\end{cases}
$$

in the hope of correcting possible errors of the model in predicting the quasi-continuum component of the dry atmospheric opacity from collision-induced-absorption. We tried fitting the dry-offsets of the 460 and $810 \mathrm{GHz}$ data from all eight pairs of SMART pixels. The value of dry-offset is clearly correlated with the pwv. All eight pairs of 460/810 pixels show similar results in Fig. 1. In this example we fit a common set of pwv and dry-offset parameters for the $460 / 810 \mathrm{GHz}$ data from each pairs of pixels. The absolute values of dry-offset are much larger when pwv is high, and nearly zero when pwv is low. Because of the large difference in the " $b$ " coefficient between the 460 and $810 \mathrm{GHz}$ bands, both of the pwv and dry-offset parameters should be determined and the dry-offset would have been zero if the atm-model were perfect.

The systematic deviation of the model from the observations indicates imperfection in the atm-model. The high frequency, high angular resolution data from astronomical observations has expanded the regime of meteorological studies, and will help to better understanding of the Earth's atmosphere, which will in turn allow astronomers to obtain more accurate submillimeter scientific data. We have already obtained more observing time on this issue and are expecting further developments in atmospheric calibration.

\section{References}

Graf, U. U., Heyminck, S., \& Michael, E. A. 2003, SPIE 4855,322

Guan, X., Stutzki, J., Graf, U. U., et al. 2012, A\& $A$ 542L, 4

Heyminck, S., Graf, U. U., Güsten R. et al. 2012, A\&A 542L, 1 